\title{
AS DIMENSÕES DA CAPACIDADE DE GESTÃO DE STAKEHOLDERS EM ORGANIZAÇÕES SEM FINS LUCRATIVOS: UM ENSAIO TEÓRICO
}

Vivian Anese $^{1}$

Carlos Costa ${ }^{1}$

Carlos Ricardo Rossetto ${ }^{2}$

${ }^{1}$ Faculdade Meridional - IMED

${ }^{2}$ Universidade do Vale do Itajaí 


\section{AS DIMENSÕES DA CAPACIDADE DE GESTÃO DE STAKEHOLDERS EM ORGANIZAÇÕES SEM FINS LUCRATIVOS: UM ENSAIO TEÓRICO}

Resumo: A forma como as organizações interagem com seus stakeholders na rotina cotidiana denota sua capacidade de gestão, especialmente levando-se em conta sua habilidade de lidar com as pressões de incerteza e escassez em seu ambiente, bem como de superar conflitos e fortalecer relações eficientes e eficazes mediante as ferramentas de que dispõem. Nesse contexto, este estudo teve como objetivo verificar se as dimensões da capacidade de gestão de stakeholders (comunicação e negociação, marketing, proatividade, formulação estratégica, recursos e stakeholder-serving), propostas por Freeman (1984) e Freeman, Harrison e Wicks (2007), para as organizações com fins lucrativos, se aplicam em organizações sem fins lucrativos. O estudo foi realizado por meio de ensaio teórico, de natureza reflexiva e interpretativa, buscando aprofundar a temática com base em articulações teóricas. Os resultados permitem verificar que as relações entre stakeholders nas duas espécies de organizações são similares, sendo possível inferir que as dimensões da capacidade de gestão propostas por Freeman e seus colaboradores, para organizações com fins lucrativos, sejam também aplicáveis às organizações sem fins lucrativos.

Palavras-chave: Teoria dos stakeholders. Dimensões da capacidade de gestão. Organizações sem fins lucrativos. ONGS.

\section{Introdução}

A capacidade de gestão está associada à forma de interação com os stakeholders na rotina cotidiana das organizações, no que se refere à sua habilidade de lidar com as pressões de incerteza e escassez no ambiente, bem como de superar conflitos e fortalecer relações eficientes e eficazes mediante as ferramentas de que dispõe. Também se relaciona às tomadas de decisão tão necessárias e ao seu gerenciamento como todo (Eskerod \& Larsen, 2018; Freeman, Harrison \& Wicks, 2007; Freeman, Harrison, Wicks, Parmar \& Colle, 2010; Gooyert, Rouwette, Kranenburg \& Freeman, 2017; Moggi, Filippi, Leardini \& Rossi, 2016; Pfeffer \& Salancik, 2003).

Nesse contexto, com o surgimento da teoria dos stakeholders aprofundada por Freeman (1984), as organizações tiveram a possibilidade de adaptar-se, sobretudo nas suas formas de relacionamentos, ou seja, tiveram que reestruturar-se para ir além da concorrência promovendo elos de acordo com seus interesses e necessidades. As vantagens trazidas pela nova teoria foram potencializadas quando Freeman, Harrison e Wicks (2007) publicaram estudos sobre stakeholders, escritos em 1984, fruto da reestrutura e aprofundamento sobre a

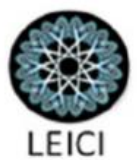


teoria e a vida prática das organizações com fins lucrativos. Tais estudos ofereceram dimensões práticas para que as organizações pudessem, por meio de uma gestão de qualidade, gerenciar as relações dos interessados, com intenção de garantir a sobrevivência, a reputação e o sucesso das empresas/organizações.

No Brasil, esse aprofundamento deu-se primeiramente por Pavão (2012), Pavão e Rossetto (2015) que aplicaram a teoria e as dimensões da capacidade de gestão dos stakeholders em estudos com cooperativas de diversos setores, organizações com fins lucrativos. A escolha desse setor para esse estudo deu-se, segundo estes autores, porque tratase de um segmento com ambiente organizacional turbulento, conflituoso, com escassos recursos e incertezas. No cenário brasileiro, não se tem notícia de outros estudos que tenham tentando aplicar as dimensões da capacidade de gestão de stakeholders, ainda que no contexto de organizações com fins lucrativos.

Considerando que a capacidade de gestão de stakeholders está relacionada diretamente com os objetivos organizacionais, e de modo especial àqueles relacionados ao capital financeiro, pensou-se que tal ideia pudesse ser aplicada em organizações que, diferentemente, mantêm as suas relações de interesses no capital humano. Assim, com os estudos de Freeman (1984), buscou-se entender a aplicabilidade dessa proposta na gestão de organizações sem fins lucrativos, visto que são empresas que também existem, persistem e precisam sobreviver.

Com base no exposto, este ensaio teórico tem como objetivo verificar se a capacidade de gestão de stakeholders criada para empresas com fins lucrativos possui aplicabilidade em organizações sem fins lucrativos, tendo como argumento central as relações do ambiente organizacional sem fins lucrativos com seus stakeholders. Desse modo, este estudo considerou as dimensões da capacidade de gestão de stakeholders - Comunicação e Negociação, Marketing, Proatividade, Formulação Estratégica, Recursos e StakeholderServing - propostas por Freeman (1984) e Freeman, Harrison e Wicks (2007), para as organizações com fins lucrativos, buscando a sua aplicabilidade em empresas sem este fim precípuo. Com tal direcionamento, a pesquisa caracteriza-se, metodologicamente, como um ensaio teórico, de natureza reflexiva e interpretativa, buscando aprofundar a temática com base em articulações teóricas.

Além desse texto introdutório que contextualiza o estudo, este ensaio teórico encontrase estruturado, de forma a contemplar os objetivos propostos, em dois outros capítulos. O primeiro, apresenta e relaciona as dimensões da capacidade de gestão de stakeholders entre as empresas com e sem fins lucrativos, divididas em seis subseções que compreendem as dimensões Comunicação e Negócio, Marketing, Formulação de Estratégias, Proatividade, Recursos e Stakeholders-Serving. Na sequência, apresenta-se as considerações finais advindas do estudo, de modo a refletir sobre a efetiva relação da gestão de stakeholders entre as espécies de empresas em foco - empresas com fins lucrativos e sem fins lucrativos.

\section{Dimensões da capacidade de gestão de stakeholders}

A teoria dos stakeholders surgiu a partir dos estudos de Freeman (1984), e faz referência a "qualquer grupo ou indivíduo que possa afetar ou ser afetado pela efetivação dos objetivos da organização" (Freeman, 1984, p. 46). Tal termo remete "àqueles que possuem uma influência ou um interesse em face da organização" (Eiró-Gomes \& Duarte, 2005, p.

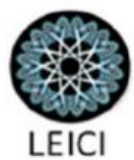


455), com a finalidade de solucionar problemas básicos de criação de valor e de comércio das organizações (Freeman et al., 2010).

Os stakeholders são identificados como pessoas, grupos, bairros, organizações, instituições, sociedades, considerando também o ambiente natural, que podem favorecer a qualificação das partes interessadas, reais ou potenciais (Mitchell, Agle, \& Wood, 1997). A visão tradicional de Freeman (1984) perante a produção da empresa/organização tem uma única preocupação, que é a de potencializar os seus fornecedores e clientes a satisfazerem suas necessidades, a fim de conquistarem o sucesso de seus negócios. Com base nas considerações do autor, várias propostas surgiram na literatura, buscando identificar a importância dos stakeholders de uma organização (Quadro 1).

\section{Quadro 1. Tipologias de classificação dos stakeholders}

\begin{tabular}{|c|c|}
\hline Autor e ano & Tipologia \\
\hline Freeman (1984) & $\begin{array}{l}\text { Aborda a teoria dos stakeholders a partir da relação entre organização e o } \\
\text { ambiente, onde os stakeholders podem afetar e serem afetados num ambiente } \\
\text { organizacional. }\end{array}$ \\
\hline $\begin{array}{l}\text { Savage, Nix, Whithead e Blair } \\
\text { (1991) }\end{array}$ & $\begin{array}{l}\text { Sugeriram avaliar o potencial de cada stakeholder quanto ao poder de } \\
\text { ameaçar ou cooperar com a organização, de modo que esta possa prevenir-se } \\
\text { e definir que atitude assumir ante cada um deles }\end{array}$ \\
\hline Donaldson e Preston (1995) & $\begin{array}{l}\text { Abordam a teoria na relação entre a organização e a sociedade sob três } \\
\text { aspectos: descritivo/empírico, instrumental e normativo. }\end{array}$ \\
\hline Mitchell et al. (1997) & $\begin{array}{l}\text { Propuseram o modelo Stakeholder Salience, no qual definiram que é preciso } \\
\text { classificar os stakeholders em termos de poder, legitimidade e urgência. Esse } \\
\text { critério de diferenciação dos grupos de stakeholders permite estabelecer } \\
\text { prioridades e definir quais interesses serão atendidos. }\end{array}$ \\
\hline Frooman (1999) & $\begin{array}{l}\text { Baseou-se nos recursos necessários para a organização e estabeleceu uma } \\
\text { matriz que relaciona o poder e a dependência entre a organização e um } \\
\text { determinado stakeholder. }\end{array}$ \\
\hline Freeman et al. (2007) & $\begin{array}{l}\text { Fazem a descrição de dez princípios concretos e sete técnicas práticas para } \\
\text { gerenciar as relações dos interessados, a fim de garantir a sobrevivência, a } \\
\text { reputação e o sucesso de uma empresa. }\end{array}$ \\
\hline Agle et al. (2008) & $\begin{array}{l}\text { Proporcionam uma reflexão sobre a gestão dos stakeholders, com destaque } \\
\text { na gerência e nos relacionamentos que estabelecem entre si. }\end{array}$ \\
\hline $\begin{array}{l}\text { Lyra, Gomes e Jacovine } \\
\text { (2009) }\end{array}$ & $\begin{array}{l}\text { Apresentam uma visão positiva da gestão dos stakeholders, que consiste na } \\
\text { aproximação entre a percepção dos stakeholders internos e externos. }\end{array}$ \\
\hline Parmar et al. (2010) & $\begin{array}{l}\text { Apresenta a teoria dos stakeholders como novidade para solucionar três } \\
\text { problemas: o da compreensão, o de conexão e o de suporte no } \\
\text { desenvolvimento da gestão. }\end{array}$ \\
\hline $\begin{array}{l}\text { Mitchell, Agle, Chrisman e } \\
\text { Spense (2011) }\end{array}$ & Abordam a teoria a partir da percepção gerencial dos stakeholders. \\
\hline $\begin{array}{l}\text { Pless, Maak e Waldman } \\
\text { (2012); Voegtlin, Patzer e } \\
\text { Scherer (2012) }\end{array}$ & $\begin{array}{l}\text { Destacam que a interação dos gestores responsáveis favorece resultados } \\
\text { positivos com os stakeholders. }\end{array}$ \\
\hline Pavão (2012) & Analisa a capacidade de gestão dos stakeholders na linha da construção civil. \\
\hline Torres (2013) & $\begin{array}{l}\text { Faz alusão à teoria dos stakeholders como processo de planejamento das } \\
\text { organizações para superação de conflitos de relacionamentos entre }\end{array}$ \\
\hline
\end{tabular}




\begin{tabular}{|c|c|}
\hline & organização e stakeholders. \\
\hline Doh e Quigley (2014) & $\begin{array}{l}\text { Destacam que os stakeholders com níveis elevados de confiança são mais } \\
\text { propensos a maior envolvimento com benefícios a longo prazo. }\end{array}$ \\
\hline Garriga (2014) & $\begin{array}{l}\text { Apresenta processos de gerência e de utilização desses numa organização em } \\
\text { relação aos stakeholders, para se relacionar com eles, identificar as } \\
\text { capacidades das partes interessadas e gerenciar relacionamentos. }\end{array}$ \\
\hline Pavão e Rossetto (2015) & $\begin{array}{l}\text { Realizaram estudos direcionados às organizações e suas relações } \\
\text { com os interesses de influências dos stakeholders a partir da capacidade de } \\
\text { gestão dos stakeholders. }\end{array}$ \\
\hline Porumbescu e Im (2015) & $\begin{array}{l}\text { Apresentam a gestão dos stakeholders com a necessidade de ser transparente } \\
\text { e confiável como estratégia de ações entre as percepções externas e internas. }\end{array}$ \\
\hline Moggi et al. (2016) & $\begin{array}{l}\text { Ressaltam que a capacidade de gestão dos stakeholders se dá a partir da } \\
\text { integração e da relação das organizações e os stakeholders. }\end{array}$ \\
\hline Gooyert et al. (2017) & $\begin{array}{l}\text { Elaboram sobre o papel dos stakeholders na tomada de decisões } \\
\text { organizacionais. }\end{array}$ \\
\hline $\begin{array}{l}\text { Schaltegger, Hörisch e } \\
\text { Freeman (2017) }\end{array}$ & $\begin{array}{l}\text { Trazem a compreensão dos casos de negócios para a sustentabilidade e como } \\
\text { podem ser criados e gerenciados pelos stakeholders. }\end{array}$ \\
\hline $\begin{array}{l}\text { Enríquez-de-Salamanca } \\
\text { (2018) }\end{array}$ & $\begin{array}{l}\text { Aborda diferentes interesses dos stakeholders na participação do processo de } \\
\text { avaliação de impacto ambiental. }\end{array}$ \\
\hline Eskerod e Larsen (2018) & $\begin{array}{l}\text { Contribuem para o debate teórico sobre o gerenciamento dos stakeholders } \\
\text { em organizações voltadas para projetos. }\end{array}$ \\
\hline
\end{tabular}

Fonte: Elaborado pelos autores (2018).

Apesar de serem vários os autores que compõem o Quadro 1 tratando sobre aspectos diversos da teoria dos stakeholders, Freeman (1984) foi quem deu maior importância ao tema sobre a capacidade de gestão de stakeholders, fazendo alusão às emergentes necessidades organizacionais da época, com ênfase nas relações gerenciais de grupos de interesse. Para o autor, a capacidade de gestão de stakeholders está associada a três níveis de análise, sendo eles: racional, transacional e processual. Quando compreendidos pelas organizações, esses três níveis de análise de gestão, interagindo nos seus interesses, destacam seu potencial de gestão e possibilitam atingir sua finalidade (Freeman, 1984).

Nesse sentido, a alta capacidade de gestão dos stakeholders é o resultado das organizações que são capazes de compreender e implementar os relacionamentos, equilibrando e mantendo os interesses com os stakeholders para que seus objetivos sejam alcançados (Freeman, 1984). Ao estruturar o material da capacidade de gestão de stakeholders, o referido autor elaborou uma tabela ilustrativa prescrevendo sete dimensões com as suas definições (Quadro 2). 
Quadro 2. Dimensões da capacidade de gestão de stakeholders propostas por Freeman (1984)

\begin{tabular}{|c|c|}
\hline $\begin{array}{c}\text { Dimensões da } \\
\text { capacidade de gestão }\end{array}$ & \multicolumn{1}{|c|}{ Conceitos prescritivos } \\
\hline $\begin{array}{c}\text { 1) Processo de } \\
\text { comunicação }\end{array}$ & $\begin{array}{l}\text { Organização com alta Capacidade de Gestão dos Stakeholders (CGS) } \\
\text { implementa processos de comunicação com os múltiplos stakeholders. }\end{array}$ \\
\hline 2) Negociação & $\begin{array}{l}\text { Organizações com alta CGS negociam explicitamente com os stakeholders } \\
\text { sobre questões críticas e buscam acordos voluntários. }\end{array}$ \\
\hline 3) Marketing & $\begin{array}{l}\text { Organizações com alta CGS difundem e aproximam o marketing para servir a } \\
\text { múltiplos stakeholders. }\end{array}$ \\
\hline $\begin{array}{c}\text { 4) Formulação } \\
\text { estratégica }\end{array}$ & $\begin{array}{l}\text { Organizações com alta CGS integram limites chave dentro do processo de } \\
\text { formulação estratégica na organização. }\end{array}$ \\
\hline 5) Proatividade & $\begin{array}{l}\text { Organizações com alta CGS são proativas, ou seja, antecipam as preocupações } \\
\text { com os stakeholders e tentam influenciar o seu ambiente. }\end{array}$ \\
\hline 6) Recursos & $\begin{array}{l}\text { Organizações com alta CGS alocam recursos de forma coerente com as } \\
\text { preocupações dos stakeholders }\end{array}$ \\
\hline 7) Stakeholder-serving & $\begin{array}{l}\text { Gestores de organizações com alta CGS pensam em termos de stakeholders a } \\
\text { que serve. }\end{array}$ \\
\hline
\end{tabular}

Fonte: Freeman (1984, p. 78-80).

Após 23 anos da elaboração desse material, Freeman, Harrison e Wicks (2007), reelaboraram outro referencial, apresentado na Figura 1, que demonstra a integração da rotina cotidiana das organizações e as preocupações dessas em relação à criação de valores com a capacidade de gestão de stakeholders. Nesse novo constructo, os autores fizeram a junção das dimensões de comunicação e negociação, devido à similaridade conceitual, aprofundando a reflexão dessas e as demais dimensões da capacidade de gestão de stakeholders (Freeman, 1984; Freeman, Harrison e Wicks, 2007).

Figura 1. Mapa conceitual das seis dimensões de capacidade de gestão de stakeholders (CGS), reestruturado por Freeman, Harrison e Wicks (2007)

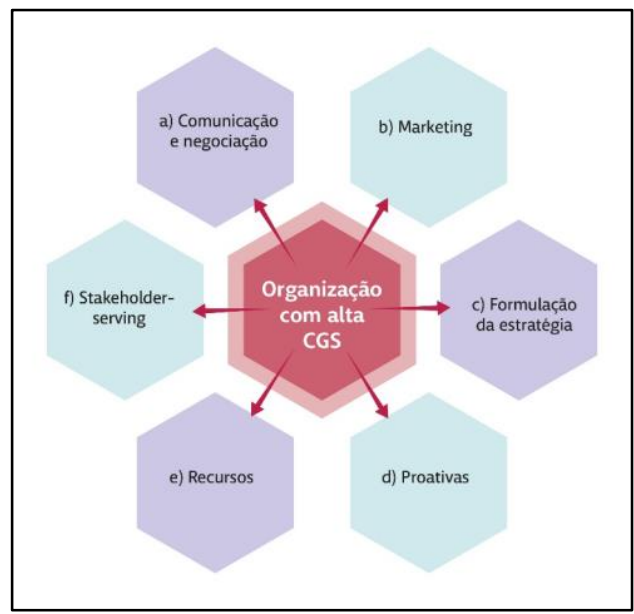

Fonte: figura elaborada pelos autores, com base nas informações de Freeman, Harrison e Wicks (2007). 
A partir do mapa conceitual das seis dimensões da capacidade de gestão de stakeholders, as subseções que seguem explanarão a conceituação reelaborada por Freeman, Harrison e Wicks (2007), bem como, as contribuições de autores encontrados nas bases de dados relacionadas em cada dimensão.

\subsection{Dimensão Comunicação e Negociação}

As organizações consideradas como de alta CGS são aquelas que idealizam e implementam, com múltiplos stakeholders, processos de comunicação, bem como, negociam sobre situações críticas e procuram combinações de interesse comum voluntariamente, ou seja, envolvem ativamente os interessados e gerenciam as próprias relações (Freeman, Harrison \& Wicks, 2007). Além disso, a organização tem o desafio de estar em uma dinâmica de atualização constante em relação aos processos gerenciais, para atender às necessidades dos stakeholders. A ideia apresentada pelos autores destaca que o voluntariado faz parte da missão da organização, por isso, seu comprometimento em satisfazer as necessidades dos seus stakeholders.

Esse processo de comunicação e negociação, analisado em organizações sem fins lucrativos, tem um suporte teórico com destaque para Waters, Burnett, Lamm e Lucas (2009) e Attouni e Mustaffa (2014), os quais afirmam que a comunicação, nessas organizações, pode se dar pelo próprio stakeholder, visto que ele é um dos meios mais eficientes para atingir os demais. Nesse mesmo sentido, Melo (2011) ressalta que um dos meios mais eficazes utilizados pelas organizações para manter contato direto com seus stakeholders é o telemarketing. Isso, pois o telemarketing é uma forma de comunicação e negociação que favorece maior interação entre a organização e seus stakeholders, uma vez que o processo de comunicação e necessidade de mostrar o que se trabalha e executa na rotina das organizações, sobretudo das sem fins lucrativos, possibilita, alcançar resultados satisfatórios (Meneghetti, 2001).

Além disso, tal dimensão de gestão usado nas organizações sem fins lucrativos deve estar contemplada na sua estratégia de gestão, precisando ser pensada na forma de divulgação e na transparência de suas ações e resultados (Lewis, 1999; Chad, Kyriazis \& Motion, 2014; Uzunoglu \& Kip, 2014). A comunicação e a negociação, por meio dessa forma estratégica, poderão influenciar diretamente na sua gestão, já que se trata de um instrumento de fácil acesso a todos.

Esse processo pode se dar de várias maneiras, incluindo o uso das mídias sociais para divulgação das ações das organizações sem fins lucrativos, que contribuem com relevante influência social (Shields, 2009; Grinstein, 2008; Bowen, 2013). Corroboram esse pensamento Carneiro, Oliveira e Torres (2011), ressaltando que a publicização das ações das organizações sem fins lucrativos é um meio de valorizar os serviços prestados utilizando a transparência, mantendo, assim, uma relação compreensiva com os stakeholders, resultando em consequências positivas de negociações segundo as necessidades e interesses.

A capacidade de gestão de stakeholders em organizações sem fins lucrativos a partir dessa dimensão de comunicação e negociação deve ser transparente. Isso, segundo Curtis et al. (2010) e Arshad, Razali e Bakar (2015), significa expandir as ações das organizações,

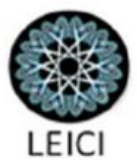


favorecendo desse modo, o reconhecimento de sua missão com planejamento, evitando lacunas e promovendo o desenvolvimento.

Além disso, o feedback é um meio de comunicação que favorece a negociação, pois tem a finalidade de compreender as necessidades dos stakeholders e ajudar no comprometimento em relação às expectativas e aos resultados planejados pela organização e, consequentemente, pelos colaboradores e doadores (Lucena, 2004). O feedback, assim, é o grande segredo dos gestores das organizações, sobretudo das organizações sem fins lucrativos, pois quanto mais mantêm contato com seus stakeholders, mais resultados positivos alcançarão nos seus objetivos.

\subsection{Dimensão Marketing}

As organizações consideradas com alta CGS são aquelas que, para servir a seus múltiplos stakeholders, expandem consideravelmente a dimensão de marketing. Para isso, investem em técnicas com o objetivo de compreender as necessidades dos stakeholders e, ainda, aproveitam a aplicação de pesquisa de marketing como uma ferramenta que possa fornecer informações para atingir a maioria dos grupos de stakeholders, que permita entendêlos e conhecer suas necessidades (Freeman, Harrison, \& Wicks, 2007).

Assim, quando não há o uso adequado das ferramentas de marketing, segundo Waters e Lord (2009), Patel e Mckeever (2014) e Sisson (2016), são afetadas, diretamente, a maneira como as ações são divulgadas, bem como o relacionamento com o público. Desse modo, as ferramentas de marketing devem ser utilizadas para atender às necessidades e às expectativas dos stakeholders das organizações sem fins lucrativos, com um maior acompanhamento e comprometimento dos gestores devido à credibilidade e à troca de informações para uma estratégia de relações consistentes (Chaves, Oikawa, Galegale, \& Azevedo, 2013).

Neste sentido, quando existe a satisfação de todos os gestores envolvidos, há também mais comprometimento de cada parte, seja da organização, seja dos stakeholders e desses entre si (Clauss \& Kesting, 2016; Sousa, 2015). A dimensão marketing, portanto, é aplicada às organizações sem fins lucrativos por ser uma ferramenta indispensável na compreensão das necessidades dos stakeholders, bem como, por ser meio viável à divulgação das ações concretizadas no ambiente organizacional e dos seus benefícios.

\subsection{Dimensão Formulação de Estratégias}

As organizações consideradas com alta CGS são aquelas que superam os obstáculos, rompendo qualquer barreira, destacando-se os processos de comunicação e a ação dos gerentes de relações públicas. Esses demonstram conhecimento sobre os gestores de produção e marketing, que têm experiências nas necessidades de clientes e fornecedores. Além disso, os gerentes conhecem sobre as preocupações do stakeholders, demonstrando responsabilidade por serem sensíveis às necessidades desses, desempenhando funções que permitam representar seus interesses dentro da organização (Freeman, Harrison \&Wicks, 2007).

Portanto, a capacidade de formulação de estratégias nas organizações sem fins lucrativos é um constante desafio. Devido ao público dessas organizações, em sua maioria, ser vulnerável e em risco social, o planejamento estratégico também se aplica, pois segundo

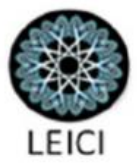


Salamon (1998), é uma alternativa para a superação das necessidades das pessoas desprovidas do atendimento do setor público.

Com isso, quando acontece de forma ativa a participação dos stakeholders-chave, que são aqueles mais importantes para a organização, no ambiente cotidiano, há possibilidades de elaboração de estratégias de utilização de recursos para sua sustentabilidade com mais agilidade. Ainda, conforme Bilodeau e Steinberg (2006), essa participação contribui para a construção de novas propostas que contribuam com a garantia dos serviços prestados pela organização.

A formulação de estratégias em qualquer organização, mas sobretudo para aquelas sem fins lucrativos, é uma dimensão indispensável, pois quando há ausência da gestão de stakeholders, Freeman (1984) e Zakhem (2008) destacam que podem ocorrer descontentamentos nas relações, gerando, segundo Lefroy e Tsarenko (2014), instabilidade e incertezas que podem interferir no planejamento. Para Pfeffer e Salancik (1978), Rossetto e Rossetto (2005) e Hillman, Withers e Collins (2009), a aplicação da formulação de estratégias para as organizações favorecem sua sobrevivência a longo prazo.

Dessa forma, compreende-se que a aplicação da dimensão formulação de estratégias em organizações sem fins lucrativos é de responsabilidade de seus gestores, pois são eles que buscam cumprir, com maior afinco, os objetivos de cada organização. Desse modo, devem fazê-lo tendo por base que a formulação de estratégias nas organizações possibilita satisfazer as necessidades de seus stakeholders. Nesse sentido, se considera a formulação de estratégias pelos gestores uma forma de contribuir para a solução de dependências externas e para o aumento da capacidade da gestão em gerir relações com o público de interesse, deixando de ser desafio, mas possibilidade e oportunidade (Freeman et al., 2010; Froelich, 1999; Frooman, 1999; Pfeffer \& Salancik, 2003).

\subsection{Dimensão Proatividade}

Em um contexto dinâmico como o empresarial, as organizações consideradas com alta CGS devem ser proativas, ou seja, eficientes, ágeis e criativas, porque se antecipam às preocupações dos stakeholders e tentam influenciar o seu ambiente. Em algumas organizações, por exemplo, acontece o investimento de recursos para intuir o que de melhor pode ser feito para satisfazer as necessidades dos clientes futuramente (Freeman, Harrison \&Wicks, 2007).

A capacidade de proatividade é entendida, nesse sentido, como uma técnica que auxilia o gestor no cumprimento da sua missão em relação aos seus stakeholders. Essa dimensão se aplica às organizações sem fins lucrativos, na medida em que o gestor que atua como influenciador e modificador do ambiente organizacional possa propiciar condições adequadas à organização com eficiência e agilidade, para estruturar-se e mudar o cenário (Pfeffer \& Salancik, 2003).

Portanto, uma das funções importantes do gestor numa organização é reduzir as incertezas e, para isso, precisa estar atento para a resolução de diferentes imprevistos nas situações cotidianas (Hillman et al., 2009; Pfeffer \& Salancik, 1978). Para que isso aconteça, o gestor necessita conhecer o contexto organizacional para propor as adaptações necessárias (Callen, Klein \& Tinkelman, 2010; Pfeffer \& Salancik, 1978; Saidel, 1991) e, ainda, construir

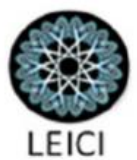


uma rede de relações, somando forças de interesse comum (Lefroy \& Tsarenko, 2014; Pfeffer $\&$ Salancik, 1978). No entanto, a eficiência de um gestor e a sua agilidade nas ações de uma organização e na manutenção de seus serviços pode gerar competição, comportamento que, segundo Gayle, Harrison e Thornton (2017), pode ser avaliado a partir de sua intensidade e considerado importante, tanto para entender e discernir, como para manter relações e construir novas.

Com isso, a capacidade de gestão na dimensão Proatividade em organizações sem fins lucrativos pode ser entendida como um serviço de habilidades e competências oferecidas para o fortalecimento e desenvolvimento do ambiente organizacional (Liao \& Huang, 2016). Quando o gestor desenvolve suas habilidades e competências com eficiência, provocando o envolvimento de todos numa organização, certamente terá resultados positivos nos objetivos organizacionais, sobretudo na redução das incertezas e na garantia de sua sobrevivência e continuidade de suas ações (Suárez \& Hwang, 2012).

\subsection{Dimensão Recursos}

As organizações consideradas com alta CGS são capazes de alocar recursos de forma coerente para atenderem as preocupações e necessidades dos seus stakeholders. Os investimentos são realizados, sobretudo, quando os grupos de interesses são importantes para o futuro e isso pode ser considerado, numa organização, como prioridade (Freeman, Harrison, \& Wicks, 2007).

Nesse contexto, a capacidade de gestão em relação à dimensão de recursos em organizações sem fins lucrativos é um dos maiores desafios, sobretudo para os gestores, pela sua responsabilidade. Tal dimensão se aplica de modo especial a essas empresas, devido à necessidade de buscarem, constantemente e das mais variadas formas, os recursos financeiros para sua sobrevivência (Tondolo, Bitencourt, \& Tondolo, 2015).

Nas empresas sem fins lucrativos, segundo Ofek (2017), há constantemente a busca por captação de recurso que é concretizada por meio de parcerias com outras organizações, similares ou não, e que fomenta uma ação primordial de criação de vínculos e benefícios, sobretudo, para quem está num contexto social de vulnerabilidade. Portanto, captar recursos é um potencial que pode ser explorado em todos os âmbitos, seja municipal, estadual, nacional ou internacional (Chad et al., 2014; Lewis, 1999; Uzunoglu \& Kip, 2014).

Para que a conquista de novas fontes de recursos seja alcançada, especialmente tratando-se de organizações sem fins lucrativos, de forma mais imediata e com maior facilidade, a gestão deve considerar a transparência das ações realizadas. Quanto maior for essa dimensão, mais credibilidade de investimentos de novos doadores e/ou mais fontes de recursos serão acessadas. Segundo Mascena, Fischmann e Boaventura (2018), isso revela um alto potencial de cooperação dos gestores na relação com seus stakeholders devido ao seu engajamento.

Vale destacar, conforme Macedo e Pinho (2006) e Lima, Ramos e Castello (2012), a importância de as organizações sem fins lucrativos manterem autonomia com fontes diversificadas de recursos, não dependendo de uma única fonte para sua sobrevivência e para manter os interesses de seus stakeholders. Uma contribuição nesse sentido, seria a alocação de recursos, sugerida por Gobo e Cadoná (2011), através da qual as ações que são executadas

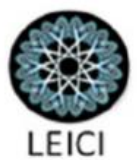


pelas organizações teriam garantidos os seus recursos necessários, podendo ser eles humanos, físicos e financeiros.

\subsection{Dimensão Stakeholders-Serving}

As organizações consideradas com alta CGS refletem esse potencial na maneira como servem seus stakeholders (satkeholders-serving). Dito de outro modo, essa dimensão diz respeito ao fato de que "tudo" o que a organização faz pressupõe-se fazê-lo de modo que favoreça o atendimento das necessidades dos seus stakeholders a longo prazo. A intenção de "como servir seu stakeholder" pode ser considerada como a "razão de ser" para as organizações que buscam manter sua missão de atender a necessidade do ambiente, evitando, assim, que a concorrência favoreça um melhor serviço. A preocupação de servir bem aos seus stakeholders fará com a que a organização sobreviva e prospere a longo prazo (Freeman, Harrison, \& Wicks, 2007).

Assim, a capacidade de gestão em relação à dimensão de stakeholder-serving se aplica às organizações sem fins lucrativos, pois essas buscam continuamente entender as necessidades dos stakeholders, satisfazê-los e servi-los com ações que são de seu interesse. Isso, por meio de serviços na área da educação, da assistência social, da saúde, da cultura, entre outras, com diversidade de opções de atividades (Freeman, 1984; Stahl, Pless, \& Maak, 2013).

Reconhecer as necessidades dos seus stakeholders e propor ações na direção de atendê-las, possibilita maior confiança e comprometimentos em níveis mais elevados da parte dos stakeholders que se entendem compreendidos em seus anseios (Doh \& Quigley, 2014). Depreende-se, assim, que cada ambiente organizacional possui peculiaridades e organiza sua gestão e faz seu planejamento com metas a objetivos positivos e próprios. Pless et al. (2012) e Voegtlin et al. (2012) destacam que os resultados positivos emergem da interação com os stakeholder. Portanto, um gestor que é capaz de compreender a necessidade de seu stakeholder, será capaz de buscar meios para que sejam supridos seus anseios.

Em organizações sem fins lucrativos, os gestores buscam os meios pelos quais seus stakeholder possam tornar-se protagonistas da própria história, agentes de transformação e pessoas livres, capazes de assumir suas escolhas com maturidade e responsabilidade. Esse resultado provoca a promoção e o desenvolvimento econômico e social não somente da organização, mas sobretudo do stakeholder que atendeu suas necessidades. Em suma, para atender as necessidades dos seus stakeholders, as organizações sem fins lucrativos precisam manter seus serviços oferecidos nas mais diversas formas. Geralmente, isso acontece a partir de uma estratégia eficiente, após o diagnóstico de uma pesquisa de satisfação, que a partir dos resultados encontrados, busca conseguir os recursos (Frooman, 1999), humanos, materiais e financeiros, para fazer a oferta segundo a demanda.

\section{Considerações finais}

Este estudo teve como objetivo verificar se a capacidade de gestão de stakeholders constatada para empresas com fins lucrativos, seria aplicável em organizações sem fins lucrativos, tendo como argumento central as relações do ambiente organizacional

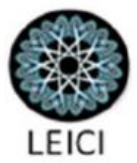


dessas últimas com seus stakeholders. Tal direcionamento, busca no aporte teórico de Freeman (1984) e Freeman, Harrison e Wicks (2007), verificar, de modo especial, as seis dimensões da capacidade de gestão consideradas pela teoria dos stakeholders, quais sejam: Comunicação e Negócio, Marketing, Formulação de Estratégicas, Proatividade, Recursos e Stakeholder-Serving.

A abordagem comparativa da capacidade de gestão das organizações sem e com fins lucrativos com seus stakeholders, para cada dimensão, permitiu concluir que:

a) Comunicação e Negócio: essa dimensão se aplica às organizações sem fins lucrativos através dos meios eletrônicos de acesso de todas as organizações, prevendo a melhoria da comunicação e negócio nas ações das referidas empresas. Isso, pois entende-se que quanto mais ágil for esse aspecto, tanto mais outras questões serão resolvidas como, por exemplo, o aumento da transparência de ações e, consequentemente, da contribuição de novos colaboradores;

b) Marketing: essa dimensão se aplica às organizações sem fins lucrativos e é de responsabilidade da gestão de tais empresas, que busca compreender e suprir as necessidades dos stakeholders;

c) Formulação de Estratégias: essa dimensão se aplica às organizações sem fins lucrativos e é realizada, de modo similar a anterior, com prioridade pelos gestores mediante as necessidades dos stakeholders e o interesse das organizações, através do comprometimento de planejamentos que possam influenciar a realização de objetivos propostos;

d) Proativas/Proatividade: essa dimensão se aplica às organizações sem fins lucrativos e é, também, considerada de responsabilidade dos gestores, devido à sua responsabilidade de se antecipar às preocupações e imprevistos em relação aos stakeholders. Isso, buscando influenciar o ambiente, causando impacto pela ousadia em melhorar suas ações de maneira eficiente;

e) Recursos: essa dimensão se aplica às organizações sem fins lucrativos e é considerada pelos seus gestores como forma de captar e alocar recursos para atender as necessidades de seus stakeholders, sejam eles recursos humanos, financeiros ou materiais;

f) Stakeholder-Serving: essa dimensão se se aplica às organizações sem fins lucrativos e concretiza-se no planejamento adequado, com atividades de desenvolvimento social e econômico quando há possibilidade, de acordo com a demanda e necessidades das pessoas.

Assim conduzida, a pesquisa mostrou que todos os elementos investigados são comuns às duas modalidades, evidenciando como os gestores de organizações sem fins lucrativos influenciam no ambiente organizacional nas quais são articuladores das relações com os stakeholders. Tal evidência é de extrema valia ao se considerar que essas organizações se encontram em um contexto cada vez mais instável e com recursos cada vez mais escassos.

Considera-se, portanto, que a capacidade de gestão de stakeholders em organizações sem fins lucrativos se dá da mesma forma que em organizações com fins lucrativos. $\mathrm{O}$ que difere é que a primeira, embora necessite de recursos para sobrevivência, investe seu capital no aspecto humano, enquanto o segundo, no seu patrimônio. Destarte, a capacidade de gestão acontece no dia a dia de cada organização e na dinâmica de cada uma viver as relações com seus stakeholders, nos mais diferentes ambientes organizacionais.

Os avanços teóricos proporcionados pelo estudo trouxeram à luz dois aspectos fundamentais e indispensáveis: os estudos de Freeman, desde 1984, e ainda os de Freeman,

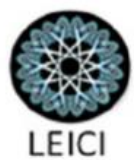


Harrison e Wicks (2007), voltados diretamente às organizações com fins lucrativos, evidenciaram por revelarem características similares, que também podem ser aplicados para as organizações sem fins lucrativos. Outra questão que se revela pertinente é que este estudo proporciona uma base literária que pode ser ainda aprofundada com novos estudos. Sobretudo, revela aos gestores de organizações sem fins lucrativos material que pode ser perscrutado e aplicado nos ambientes dessas organizações na construção de relações que favoreçam interesses e atendam necessidades, de acordo com as particularidades de cada uma.

$\mathrm{O}$ fator limitante do estudo evidencia-se pelo fato de ser um estudo parcial e fundamentado em poucos autores natos e/ou específicos da área das organizações sem fins lucrativos com dados em profundidade sobre o tema dos stakeholders. Portanto, sugere-se que estudos futuros possam aprofundar as diferenças e as semelhanças na gestão de organizações com fins lucrativos e em organizações sem fins lucrativos. Ainda, pode-se fazer um estudo bibliométrico nas bases de dados considerados de alto índice, para ver se estudos sobre as dimensões da capacidade de gestão em organizações sem fins lucrativos já foram aplicados em outros países.

\section{Referências}

Agle, B. R., Donaldson, T., Freeman, E., Jensen, M., Mitchell, R. K., \& Wood, D. J. (2008). Dialogue: toward superior stakeholder theory. Business Ethics Quarterly, 18,153-190. https://doi.org/10.5840/beq200818214

Arshad, R., Razali, W. A. A.W. M., \& Bakar, N. A. (2015). Catch the warning signals: the fight against fraud and abuse in nonprofit organisations. Procedia Economics and Finance, 28,114-120. https://doi.org/10.1016/S2212-5671(15)01089-8

Attouni, M. A. K., \& Mustaffa, C. S. (2014). How do nonprofit organizations in Libya adopt and use social media to communicate with the society. Procedia social and Behavioral Sciences, 155, 92-97. https://doi.org/10.1016/j.sbspro.2014.10.262

Bilodeau, M., \& Steinberg, R. (2006). Donative nonprofit organizations. In S. C. Kolm \& J. M. Ythier (Eds.). Handbook of the economics of giving, altruism and reciprocity (v. 4, cap.19, p. 1272-1307). Indianopolis: Elsevier. https://doi.org/10.1016/S1574-0714(06)02019$\underline{7}$

Bowen, S. A. (2013). Using classic social media cases to distill ethical guidelines for digital engagement. Journal of Mass Media Ethics, 28(2), 119- 133.

https://doi.org/10.1080/08900523.2013.793523

Callen, J. L., Klein, A., \& Tinkelman, D. (2010). The contextual impact of nonprofit board composition and structure on organizational performance: agency and resource dependence perspectives. Voluntas: International Journal of Voluntary and Nonprofit Organizations, 21(1), 101-125. DOI 10.1007/s11266-009-9102-3

Carneiro, A.F., Oliveira, D. L., \& Torres, L.C. (2011). Accountability e prestação de contas das organizações do terceiro setor: uma abordagem à relevância da contabilidade. Sociedade, Contabilidade e Gestão, 6(2), 90-105.

Chad, P., Kyriazis, E., \& Motion, J. (2014). Bringing marketing into nonprofit organizations: a managerial nightmare. Australasian Marketing Journal, 22, 342-349.

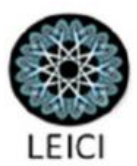


https://doi.org/10.1016/j.ausmj.2014.09.003

Chaves, E. C. J., Oikawa, R.,Galegale, N. V., \& Azevedo, M. M. (2013). Avaliação da gestão de stakeholders em implantações de projetos de sistemas em serviços. In VIII Whorkshop de Pós-Graduação e Pesquisa do Centro Paula Souza (páginas). São Paulo, SP.

Clauss, T., \& Kesting, T. (2016). How businesses should govern knowledge - intensive collaborations with univesities: na empirical investigation of university professor. Industrial Marketing Management, 62, 185-198. https://doi.org/10.1016/j.indmarman.2016.09.001 Curtis, L., Edwards, C., Fraser, K. L., Gudelsky, S., Holmquist, J., Thornton, K., \& Sweetser, K. D. (2010). Adoption of social media for public relations by nonprofit organizations. Public Relations Review, 36, 90-92. https://doi.org/10.1016/j.pubrev.2009.10.003

Doh, J. P., \& Quigley, N. R. (2014). Responsible leadership and stakeholder management: influence pathways and organizational outcomes. The academy of Management Perspectives, 28(3), 255-274. doi:10.5465/amp.2014.0013

Donaldson, T., \& Preston, L. E. (1995). The stakeholder theory of the corporation: concepts, evidence, and implications. Academy of Management Review, 20(1), 65-91.

DOI: $10.2307 / 258887$

Eiró-Gomes, M.; Duarte, J. Que públicos para as relações públicas? Actas Dos III Sopcom, VI Lusocom e II Ibérico, Vol. II, Comissão Editorial da Universidade de Beira Interior, Covilhã, Portugal, p. 453-461, 2005.

Eskerod, P., \& Larsen, T. (2018). Advancing project stakeholder analysis by the concept 'shadows of the context'. International Journal of Project Management, 36, 161-169. https://doi.org/10.1016/j.ijproman.2017.05.003

Enríquez-De-Salamanca, A. (2018). Stakeholders' manipulation of environmental impact assessment. Environmental Impact Assessment Review, 68, 10-18.

https://doi.org/10.1016/j.eiar.2017.10.003

Freeman, R. E. (1984). Strategic management: a stakeholder approach. Boston: Pitman.

Freeman, R. E., Harrison, J. S., \& Wicks, A. (2007). Managing for stakeholder: survival, reputation, and sucess. London: Yale University Press.

Freeman, R. E., Harrison, J. S., Wicks, A. C., Parmar, B., \& Colle, S. (2010). Stakeholder theory: the state of the art. New York: Cambridge University Press.

Froelich, K. A. (1999). Diversification of revenues strategies: evolving resource in nonprofit organizations. Nonprofit and Voluntary Sector Quarterly, 28(3), 246-268

Frooman, J. Stakeholders influence strategies. (1999). Academy of Management Review, 24(2), 191-205. DOI: 10.2307/259074

Gayle, P. G., Harrison, T. D., \& Thornton, J. (2017). Entry, donor Market size, and competitive conduct among nonprofit firms. International Journal of Industrial Organization, 50, 294-318. https://doi.org/10.1016/j.ijindorg.2016.10.003

Garriga, E. (2014). Beyond stakeholder utility function: stakeholder capability in the value creation process. Journal of Business Ethics, 120(4), 489-507.

https://doi.org/10.1007/s10551-013-2001-y

Grinstein, A. (2008). The relationships between market orientation and alternative strategic orientations. European Journal of Marketing, 42, 115-134.

https://doi.org/10.1108/03090560810840934 
Gobo, R.V., \& Cadoná, C. (2011). Ferramenta de alocação de recursos humanos em projetos. Canoas - RS: Curso de Ciência da Computação da Universidade Luterana do Brasil (ULBRA).

Gooyert, V., Rouwette, E., Kranenburg, H. V., \& Freeman, E. (2017). Reviewing the role of stakeholders in Operational Research: a stakeholder theory perspective. European Journal of Operational Research. Elsevier, 262(2), 402-410. https://doi.org/10.1016/j.ejor.2017.03.079 Hillman, A. J., Withers, M. C., \& Collins, B. J. (2009). Resource dependence theory: a review. Journal of Management, 35(6), 1404-1427. DOI: 10.1177/0149206309343469 Lefroy, K., \& Tsarenko, Y. (2014). Dependence and effectiveness in the nonprofit-corporate alliance: the mediating effect of objectives achievement. Journal of Business Research, 67, 1959-1966. https://doi.org/10.1016/j.jbusres.2013.11.003

Lewis, L. K. (1999). Disseminating information and soliciting input during planned organisational change: implementers' targets, sources, and channels for communicating. Management Communication Quarterly, 13(1), 43-75. https://doi.org/10.1177/0893318999131002

Liao, K., \& Huang, I. (2016). Impact of vision, strategy, and human resource on nonprofit organization service performance. Procedia Social and Behavioral Sciences, 224, 20-27. https://doi.org/10.1016/j.sbspro.2016.05.395

Lima, M. A. S., Ramos, N. R. G., \& Castello, R. N. (2012). Organizações do terceiro setor e a teoria da dependência de recursos: um estudo de Benevides, na Amazônia brasileira, e Ilha de Santiago, em Cabo Verde, na África. In Congresso Transformare (1-17). Paris.

Lucena, M. D. S. (2004). Planejamento estratégico e gestão do desempenho por resultados. São Paulo: Atlas.

Lyra, M. G., Gomes, R. C., \& Jacovine, L. A. G. (2009). O papel dos stakeholders na sustentabilidade da empresa: contribuições para construção de um modelo de análise. Revista de Administração Contemporânea, 13, 39-52.

Macedo, I. M., \& Pinho, J. C. (2006). The relationship between resource dependence and market orientation: the specific case of non-profit organizations. European Journal of Marketing, 40(5), 533-553. DOI: 10.1108/03090560610657822

Mascena, K. M. C., Fischmann, A. A., \& Boaventura, J. M. G. (2018). Priorização de stakeholders em empresas que divulgam relatórios GRI no Brasil. Brazilian Business Review, 15(1), 17-32.

Melo, T. G. (2011). Insalubridade no teleatendimento e telemarketing (Monografia). Faculdade de Direito, Universidade de Presidente Antonio Carlos (UNIPAC), Barbacena. Meneghetti, S. B. (2001). Comunicação e marketing: fazendo a diferença no dia a dia das organizações da sociedade civil. São Paulo: Global.

Mitchel, R.K., Agle, B. R., \& Wood, D. J. (1997). Toward a theory os stakeholder identification and salience: defining the principle of who and what really counts. Academy of Management Review, 22(4), 856-886. DOI: 10.2307/259247

Mitchell, R.K., Agle, B. R., Chrisman, J. J., \& Spense, L. J. (2011). Toward a theory of stakeholder salience in family firms. Business Ethics Quarterly, 21(2), 235-255.

https://doi.org/10.5840/beq201121215

Moggi, S., Filippi, V., Leardini, C., \& Rossi, G. (2016). Accountability for a place in heaven: a stakeholders'portrait in Verona's confraternities. Accounting History, 21(2/3), 236-262. 
https://doi.org/10.1177/1032373216643078

Ofek, Y. (2017). Evaluating social exclusion interventions in university - community partnerships. Evaluation and Program Planning, 60, 46-55.

https://doi.org/10.1016/j.evalprogplan.2016.09.004

Parmar, B. L., Freeman, R. E., Harrison, J. S., Wicks, A. C., Purnell, L., \& Colle, S. (2010). Stakeholder theory: the state of the art. Academy of Management Annals, 4(1), 403-445. https://doi.org/10.1080/19416520.2010.495581

Patel, S. J., \& Mckeever, B. (2014). Health nonprofits online: the use of frames and frames and stewardship strategies to increase stakeholders involvement. International Journal of Nonprofit and Voluntary Sector Marketing, 19(4), 224-238. DOI: 10.1002/nvsm.1507 Pavão, Y. M. P. (2012). A capacidade de gestão dos stakeholders e o ambiente organizacional: relações e impacto no desempenho de cooperativas do Brasil (Tese de Doutorado). Faculdade de Administração, Universidade do Vale do Itajaí (Univali). Biguaçu, SC.

Pavão, Y. M. P., \& Rossetto, C. R. (2015). Stakeholder management capability and performance in Brazilian cooperatives. Review of Business Management, 17(55), 870-889. http://dx.doi.org/10.7819/rbgn.v17i55.2125

Pfeffer, J., \& Salancik, G. R. (1978). The external control of organizations: a resource dependence perspective. New York: Harper and Row.

Pfeffer, J., \& Salancik, G. R. (2003). The external control of organizations. A resource dependence perspective. New York: Harper and Row.

Pless, N., Maak, T., \& Waldman, D. A. (2012). Different approaches toward doing the right thing: Mapping the responsibility orientations of leaders. Academy of Management

Perspectives, 26(4), 51-65.

doi:10.5465/amp.2012.0028

Porumbescu, G., \& Im, T. (2015). Using transparency to reinforce responsibility and responsiveness. In J. Perry \& R. Christensen, The handbook of public administration (3th ed., pp. 120-36). San Francisco, CA: Wiley.

Rossetto, C. R., \& Rossetto, A. M. (2005). Teoria institucional e dependência de recursos na adaptação organizacional: uma visão complementar. Revista de Administração de Empresas, 4(1), 1-22.

Saidel, J. R. (1991). Resource interdependence: the relationship between state agencies and nonprofi t organizations. Public Administration Review, 5(6), 543-53.

DOI: $10.2307 / 976605$

Salamon, L. M. (1998). A emergência do terceiro setor: uma revolução associativa global. Revista de Administração, 33(1), 5-11.

Savage, G. T., Nix, T.W., Whithead, C. J., \& Blair, J. D. (1991). Strategies for assessing and managing organizational stakeholders. Academy of Management Executive, 5(2), 65-75.

Shields, P.O. (2009). Young adult volunteers: recruitment appeals and other marketing considerations. Journal of Nonprofit e Public Sector Marketing, 21, 139-159.

https://doi.org/10.1080/10495140802528658

Sisson, D. (2016). Control mutuality, social media, and organization - public relationships: a study of local animal welfare organizations donors. Public Relations Review, 42(4), p. 1-11. 
Sousa, C. (2015). Evaluating progress towards sustainable development: a critical examination of a decade-long university-nonprofit collaboration. Current Opinion in Environmental Sustainability, 17, 10-21. https://doi.org/10.1016/j.cosust.2015.06.008 Suárez, D. F., \& Hwang, H. (2012). Resource contraints or cultural conformity? Nonprofit relationships with businesses. Voluntas: International Journal of Voluntary and Nonprofit Organizations, 24(3), 581- 605. https://doi.org/10.1007/s11266-012-9267-z

Stahl, G. K., Pless, N. M., \& Maak, T. (2013). Responsible global leadership. In M. E. Mendenhall, J. Osland, A. Bird, G. R. Oddou, M. L. Maznevski, \& G. K. Stahl (Eds.), Global leadership: research, practice, and development (pp.240-259). New York: Routledge.

Schaltegger, S., Hörisch, J., \& Freeman, E. (2017). Business cases for sustainability: a stakeholder theory perspective. Organization \& Environment, 1-22.

https://doi.org/10.1177/1086026617722882

Tondolo, R. R. P., Bitencourt, C. C., \& Tondolo, V. A. G. (2015). Third sector management: as analysis from the mobolizing dimension of organizational social capital. In Anais for 14th International Vongress of International Association on Public a Nonprofit Marketing. Vitória, ES.

Torres, L. H. (2013). Teoria do stakeholder: um estudo da aplicação do princípio de equidade do stakeholder (Dissertação de Mestrado). Faculdade de Administração, Universidade Federal do Rio Grande do Sul, Porto Alegre, RS.

Uzunoglu, E., \& Kip, S. M. (2014). Building relationships through websites: a contente analysis of Turkish environmental nonprofit organizations (NPO) websites. Public Relations Review, 40, 113-115. https://doi.org/10.1016/j.pubrev.2013.06.001

Voegtlin, C., Patzer, M., \& Scherer, A. G. (2012). Responsible leadership in global business: A new approach to leadership and its multilevel outcomes. Journal of Business Ethics, 105(1) 1-16. https://doi.org/10.1007/s10551-011-0952-4

Waters, R. D., Burnett, E., Lamm, A., \& Lucas, J. (2009). Engaging stakeholders through social networking: how nonprofit organizations are using facebook. Public Relations Review, 35(2), 102-106. https://doi.org/10.1016/j.pubrev.2009.01.006

Waters, R. D., \& Lord, M. (2009). Examining how advocacy groups build relationships on the internet. International Journal of nonprofit and Voluntary Sector Marketing, 14(3), 231 241. DOI: $10.1002 /$ nvsm.354

Zakhem, A. (2008). Stakeholder management capability: a discourse theoretical approach. Journal of Business Ethics, 79(4), 395-405. https://doi.org/10.1007/s10551-007-9405-5 